\title{
ALMOST SUMMABLE SEQUENCES
}

\author{
J. P. KING
}

1. Introduction. Let $A=\left(a_{n k}\right)$ be an infinite matrix of complex numbers and let $x=\left\{x_{k}\right\}$ be a sequence of complex numbers. The sequence $\sigma=\left\{\sigma_{n}\right\}$ defined by

$$
\sigma_{n}=\sum_{k=0}^{\infty} a_{n k} x_{k}
$$

is called the $A$-transform of $x$ whenever the series converges for $n=0,1, \cdots$. The sequence $x$ is said to be $A$-summable to $y$ if $\left\{\sigma_{n}\right\}$ converges to $y . A$ is called conservative if $x \in c$ implies $\sigma \in c$, where $c$ is the linear space of convergent sequences. $A$ is called regular if it is conservative and preserves the limit of each convergent sequence.

In the theory of summability and its applications one is usually interested in conservative or regular matrices. It is the object of this paper to introduce a slightly more general class of matrices than the conservative or regular matrices and to give certain illustrations of this notion.

2. Definitions. Let $m$ denote the linear space of bounded sequences. A sequence $x \in m$ is said to be almost convergent and $s$ is called its generalized limit if each Banach limit [3] of $x$ is $s$. The class $f$ of almost convergent sequences was introduced by Lorentz [3], who proved that a sequence $x=\left\{x_{k}\right\}$ is almost convergent if and only if

$$
\lim _{p \rightarrow \infty} \frac{x_{n}+x_{n+1}+\cdots+x_{n+p-1}}{p}=s
$$

uniformly in $n$.

A convergent sequence is almost convergent and its limit and its generalized limit are identical. Lorentz proved that $f$ is a closed linear subspace of $m$ in the usual topology.

Definition 2.1. A sequence $x$ is said to be almost convergent to $s$ if $x \in f$ and $s$ is the generalized limit of $x$.

Definition 2.2. A sequence $x$ is said to be almost $A$-summable if the $A$-transform of $x$ is almost convergent. It is said to be almost $A$-summable to $s$ if the A-transform of $x$ is almost convergent to $s$.

Definition 2.3. The matrix $A$ is said to be almost conservative if

Received by the editors March 9, 1966. 
$x \in c$ implies that the $A$-transform of $x$ is almost convergent. $A$ is said to be almost regular if the A-transform of $x$ is almost convergent to the limit of $x$ for each $x \in c$.

In the sequel the following notation is used:

(i) $C$ denotes the complex numbers,

(ii) $Z$ denotes the integers,

(iii) $Z^{+}$denotes the positive integers,

(iv) If $x=\left\{x_{k}\right\}$ is an element of $c$, then $\|x\|$ is defined by

$$
\|x\|=\sup \left\{\left|x_{k}\right|: k \in Z\right\},
$$

(v) The linear space of all continuous linear functionals on $c$ is denoted by $c^{\prime}$,

(vi) If $f \in c^{\prime}$ then $\|f\|$ is defined by

$$
\|f\|=\sup \{|f(x)|:\|x\|=1\} .
$$

It is well known that the functionals defined by (iv) and (vi) of the above are norms for $c$ and $c^{\prime}$, respectively, and that the resulting normed spaces are complete.

\section{Theorems.}

TheOREM 3.1. The matrix $A=\left(a_{n k}\right)$ is almost conservative if and only if

(3.1) $\sup \left\{\sum_{k=0}^{\infty} \frac{1}{p}\left|\sum_{j=n}^{n+p-1} a_{j k}\right|: p \in Z^{+}\right\}<\infty, \quad n=0,1, \cdots$,

(3.2) there exists $\alpha_{k} \in C, k=0,1, \cdots$, such that

$$
\lim _{p \rightarrow \infty} \frac{1}{p} \sum_{j=n}^{n+p-1} a_{j k}=\alpha_{k}
$$

uniformly in $n$, and

(3.3) there exists $\alpha \in C$ such that

$$
\lim _{p \rightarrow \infty} \frac{1}{p} \sum_{j=n}^{n+p-1} \sum_{k=0}^{\infty} a_{j k}=\alpha,
$$

uniformly in $n$.

Proof. Suppose that $A$ is almost conservative. Fix $n \in Z$. Let

$$
t_{p n}(x)=\frac{1}{p} \sum_{j=n}^{n+p-1} \sigma_{j}(x),
$$

where 


$$
\sigma_{j}(x)=\sum_{k=0}^{\infty} a_{j k} x_{k}
$$

It is clear that $\sigma_{j} \in c^{\prime}, j=0,1, \cdots$. Hence $t_{p n} \in c^{\prime}, p=1,2, \cdots$. Since $A$ is almost conservative,

$$
\lim _{p \rightarrow \infty} t_{p n}(x)=t(x)
$$

uniformly in $n$. It follows that $\left\{t_{p n}(x)\right\}$ is bounded for $x \in c$ and fixed $n$. Hence, $\left\{\left\|t_{p n}\right\|\right\}$ is bounded by the uniform boundedness principle.

For each $r \in Z^{+}$, define the sequence $y=y(n, p)$ by

$$
\begin{aligned}
y_{k} & =\operatorname{sgn} \sum_{j=n}^{n+p-1} a_{j k}, & & 0 \leqq k \leqq r \\
& =0, & & r<k .
\end{aligned}
$$

Then $y \in c,\|y\|=1$, and

$$
\left|t_{p n}(y)\right|=\frac{1}{p} \sum_{k=0}^{r}\left|\sum_{j=n}^{n+p-1} a_{j k}\right| .
$$

Hence

$$
\left|t_{p n}(y)\right| \leqq\left\|t_{p n}\right\|\|y\|=\left\|t_{p n}\right\| \text {. }
$$

Therefore

$$
\frac{1}{p} \sum_{k=0}^{\infty}\left|\sum_{j=n}^{n+p-1} a_{j k}\right| \leqq\left\|t_{p n}\right\|
$$

so that (3.1) follows.

Let the sequences $e$ and $e_{k}, k=0,1, \cdots$, be defined by $e=(1,1, \cdots, 1, \cdots)$ and $e_{k}=(0, \cdots, 0,1,0, \cdots)$ where the last 1 is in the $k$ th position. Since $e$ and $e_{k}$ are convergent sequences, $k=0,1, \cdots, \lim t_{p n}(e)$ and $\lim t_{p n}\left(e_{k}\right)$ must exist uniformly in $n$. Hence (3.2) and (3.3) must hold.

Now assume that (3.1), (3.2), and (3.3) hold. Fix $n \in Z$ and $x \in c$. Then

$$
\begin{aligned}
t_{p n}(x) & =\frac{1}{p} \sum_{j=n}^{n+p-1} \sum_{k=0}^{\infty} a_{j k} x_{k} \\
& =\frac{1}{p} \sum_{k=0}^{\infty} \sum_{j=n}^{n+p-1} a_{j k} x_{k}
\end{aligned}
$$

so that 


$$
\left|t_{p n}(x)\right| \leqq \frac{1}{p} \sum_{k=0}^{\infty}\left|\sum_{j=n}^{n+p-1} a_{j k}\right|\|x\| .
$$

Therefore, $\left|t_{p n}(x)\right| \leqq K_{n}\|x\|$ by (3.1), where $K_{n}$ is a constant independent of $p$. Hence $t_{p n} \in c^{\prime}, p=1,2, \cdots$, and the sequence $\left\{\left\|t_{p n}\right\|\right\}$ is bounded for each $n \in Z^{+}$. (3.2) and (3.3) imply that $\lim t_{p n}(e)$ and $\lim t_{p n}\left(e_{k}\right)$ exist for $n=0,1, \cdots$, and $k=0,1, \cdots$. Since $\left\{e, e_{0}, e_{1}, \cdots\right\}$ is a fundamental set in $c$ it follows from an elementary result of functional analysis (see, e.g., $[2$, p. 252]) that

$$
\lim _{p \rightarrow \infty} t_{p n}(x)=t_{n}(x)
$$

exists and $t_{n} \in c^{\prime}$. Therefore, $t_{n}$ has the form [2, p. 205]

$$
t_{n}(x)=\beta\left[t_{n}(e)-\sum_{k=0}^{\infty} t_{n}\left(e_{k}\right)\right]+\sum_{k=0}^{\infty} x_{k} t_{n}\left(e_{k}\right),
$$

where $\beta=\lim x_{k}$. But $t_{n}(e)=\alpha$ and $t_{n}\left(e_{k}\right)=\alpha_{k}, k=0,1, \cdots$, by (3.3) and (3.2), respectively. Hence

$$
\lim _{p \rightarrow \infty} t_{p n}(x)=t(x)
$$

exists for each $x \in c$ and $n=0,1, \cdots$, with

$$
t(x)=\beta\left[\alpha-\sum_{k=0}^{\infty} \alpha_{k}\right]+\sum_{i=0}^{\infty} \alpha_{k} x_{k} .
$$

Since $t_{p n} \in c^{\prime}$ for each $p$ and $n$, it has the form

$$
t_{p n}(x)=\beta\left[t_{p n}(e)-\sum_{k=0}^{\infty} t_{p n}\left(e_{k}\right)\right]+\sum_{k=0}^{\infty} x_{k} t_{p n}\left(e_{k}\right) .
$$

It is easy to see from (3.4) and (3.5) that the convergence of $\left\{t_{p n}(x)\right\}$ to $t(x)$ is uniform in $n$, since $t_{p n}(e) \rightarrow \alpha$ and $t_{p n}\left(e_{k}\right) \rightarrow \alpha_{k}$ uniformly in $n$. Therefore, $A$ is almost conservative and the theorem is proved.

THEOREM 3.2. The matrix $A$ is almost regular if and only if

(3.6) $\sup \left\{\sum_{k=0}^{\infty} \frac{1}{p}\left|\sum_{j=n}^{n+p-1} a_{j k}\right|: p \in Z^{+}\right\}<\infty, \quad n=1,2, \cdots$,

(3.7) $\lim _{p \rightarrow \infty} \frac{1}{p} \sum_{j=n}^{n+p-1} a_{j k}=0, \quad$ uniformly in $n, k=0,1, \cdots$,

and 


$$
\lim _{p \rightarrow \infty} \frac{1}{p} \sum_{j=n}^{n+p-1} \sum_{k=0}^{\infty} a_{j k}=1, \quad \text { uniformly in } n .
$$

Proof. Suppose that $A$ is almost regular. Then $A$ is almost conservative so that (3.6) must hold by Theorem 3.1. (3.7) and (3.8) must hold since the $A$-transforms of the sequences $e_{k}, k=0,1, \cdots$, and $e$ must be almost convergent to 0 and 1 , respectively.

Suppose that (3.6), (3.7), and (3.8) hold. Then $A$ is almost conservative by Theorem 3.1. Therefore, $\lim t_{p n}(x)=t(x)$ uniformly in $n$ for each $x \in c$. The representation (3.4) gives $t(x)=\lim x_{k}$. Hence, $A$ is almost regular. This proves the theorem.

It is interesting to compare Theorem 3.2 with the well known Silverman-Toeplitz Theorem which gives necessary and sufficient conditions that $A$ be a regular matrix. The Silverman-Toeplitz conditions are

$$
\begin{gathered}
\sup _{n}\left\{\sum_{k=0}^{\infty}\left|a_{n k}\right|: n \in Z\right\}<\infty, \\
\left\{a_{n k}: n \in Z\right\} \text { converges to } 0, \quad k=0,1, \cdots,
\end{gathered}
$$

and

$$
\left\{\sum_{k=0}^{\infty} a_{n k}: n \in Z\right\} \text { converges to } 1 .
$$

It is clear that (3.9) implies (3.6). The criteria of Theorem 3.2 which correspond to (3.10) and (3.11) are that the sequences $\left\{a_{n k}: n \in Z\right\}$ and $\left\{\sum_{k=0}^{\infty} a_{n k}: n \in Z\right\}$ shall be almost convergent to 0 , $k=0,1, \cdots$, and to 1 , respectively.

In the applications of summability theory to function theory it is important to know the region in which the sequence of partial sums of the geometric series is $A$-summable to $1 /(1-z)$ for a given matrix $A$. The following theorem is helpful in determining the region in which the sequence of partial sums of the geometric series is almost $A$-summable to $1 /(1-z)$.

Theorem 3.3. Let $A$ be a matrix such that (3.8) holds. The sequence of partial sums of the geometric series is almost A-summable to $1 /(1-z)$ if and only if $z \in \Omega$ where

$$
\Omega=\left\{z: \lim _{p \rightarrow \infty} \frac{1}{p} \sum_{j=n}^{n+p-1} \sum_{k=0}^{\infty} a_{j k} z^{k}=0 \text { uniformly in } n\right\} .
$$

Proor. Let $\left\{s_{k}(z)\right\}$ denote the sequence of partial sums of the geo- 
metric series. Then

$$
\begin{aligned}
t_{p n} & =\frac{1}{p} \sum_{j=n}^{n+p-1} \sum_{k=0}^{\infty} a_{j k} s_{k}(z) \\
& =\frac{1}{p} \sum_{j=n}^{n+p-1} \sum_{k=0}^{\infty} a_{j k} \frac{1-z^{k+1}}{1-z} .
\end{aligned}
$$

Hence,

$$
\lim _{p \rightarrow \infty} t_{p n}=\frac{1}{1-z}-\lim _{p \rightarrow \infty} \frac{z}{p(1-z)} \sum_{j=n}^{n+p-1} \sum_{k=0}^{\infty} a_{j k} z^{k} .
$$

Therefore

$$
\lim _{p \rightarrow \infty} t p_{n}=\frac{1}{1-z}
$$

uniformly in $n$, if and only if $z \in \Omega$.

4. Applications. The Euler matrix $E(r)$ is defined by

$$
\begin{aligned}
b_{n k} & =\left(\begin{array}{l}
n \\
k
\end{array}\right) r^{k}(1-r)^{n-k}, \quad 0 \leqq k \leqq n \\
& =0, \quad n<k .
\end{aligned}
$$

It is known that $E(r)$ is regular if and only if $0<r \leqq 1[1]$.

THEOREM 4.1. Let $r \neq 0$ be a complex number. The sequence of partial sums of the geometric series is almost $E(r)$-summable to $1 /(1-z)$ if and only if $z \in \Omega_{r}$ where

$$
\Omega_{r}=\{z:|z-(1-1 / r)| \leqq 1 /|r|, z \neq 1\} .
$$

Proof. Since $\sum_{k=0}^{\infty} b_{n k}=1$, (3.8) holds for $E(r)$. Hence, by Theorem 3.3 it is sufficient to show that $\Omega=\Omega_{r}$. In this case

$$
\begin{aligned}
\sum_{j=n}^{n+p-1} \sum_{k=0}^{\infty} b_{j k} z^{k} & =\sum_{j=n}^{n+p-1} \sum_{k=0}^{j}\left(\begin{array}{l}
j \\
k
\end{array}\right) r^{k}(1-r)^{j-k} z^{k} \\
& =\sum_{j=n}^{n+p-1}(1-r+r z)^{j} \\
& =(1-r+r z)^{n} \frac{1-(1-r+r z)^{p}}{1-(1-r+r z)} .
\end{aligned}
$$

Therefore, $z \in \Omega$ if and only if $|1-r+r z| \leqq 1, z \neq 1$. Hence, $\Omega=\Omega_{r}$.

In order that the sequence of partial sums be $E(r)$-summable to 
$1 /(1-z)$ it is necessary that $z$ lie strictly inside the disk $\Omega_{\text {r }}$.

It is known [1] that $E(r)$ is regular if and only if $0<r \leqq 1$. It is natural to inquire whether or not there exist values of $r$ for which $E(r)$ is almost regular but not regular. Unfortunately, this is not the case.

THEOREM 4.2. $E(r)$ is almost regular if and only if it is regular.

Proof. If $0<r \leqq 1$ then $E(r)$ is regular and, hence, almost regular. Suppose $E(r)$ is almost regular. Since an almost convergent sequence is bounded, (3.7) will hold only if $|1-r|<1$. If $\Delta=\{z:|z|<1\}$, then $\Delta \subset \Omega_{r}$, since the geometric series converges to $1 /(1-z)$ if $z \in \Delta$. Since $\Omega_{r}$ is a disk with center $(1-1 / r)$ whose boundary passes through $z=1$, it is evident that $1-1 / r \leqq 0$. Hence, $r \leqq 1$. This condition together with $|1-r|<1$ implies $0<r \leqq 1$. Therefore, $E(r)$ is regular.

The following result provides an example of a matrix which is almost regular but not regular.

Theorem 4.3. Let $C=\left(c_{n k}\right)$ be defined by

$$
\begin{aligned}
c_{n k} & =\frac{1}{n+1}\left[1+(-1)^{n}\right], & & 0 \leqq k \leqq n, \\
& =0, & & n<k .
\end{aligned}
$$

Then $C$ is almost regular but not regular.

Proof. $C$ is not regular since

$$
\lim _{n \rightarrow \infty} \sum_{k=0}^{\infty} c_{n k}
$$

does not exist. It is easy to see that the hypotheses of Theorem 3.2 hold for the matrix $C$ so that $C$ is almost regular.

The author is indebted to the referee for some helpful suggestions. Added in proof. It has been pointed out to the author, by Mr. Jerry D. Badger, that (3.1) and (3.6) can be replaced by the more natural (3.9).

\section{REFERENCES}

1. R. P. Agnew, Euler transformations, Amer. J. Math. 66 (1944), 318-338.

2. L. V. Kantorovich and G. P. Akilov, Functional analysis in normed spaces, Macmillan, New York, 1964.

3. G. G. Lorentz, A contribution to the theory of divergent sequences, Acta. Math. 80 (1948), 167-190.

\section{LEHIGH UNIVERSITY}

